\title{
Adding a Diversity Mechanism to a Simple Evolution Strategy to Solve Constrained Optimization Problems
}

\author{
Efrén Mezura-Montes \& Carlos A. Coello Coello \\ CINVESTAV-IPN (Evolutionary Computation Group) \\ Departamento de Ingeniería Eléctrica, Sección de Computación \\ Av. IPN No. 2508. Col. San Pedro Zacatenco México D.F. 07300, MÉXICO \\ emezura@computacion.cs.cinvestav.mx \\ ccoello@cs.cinvestav.mx
}

\begin{abstract}
In this paper, we propose the use of a Simple Evolution Strategy (SES) (i.e., a $(1+\lambda)$-ES with self-adaptation that uses three tournament rules based on feasibility) coupled with a diversity mechanism to solve constrained optimization problems. The proposed mechanism is based on multiobjective optimization concepts taken from an approach called the Niched-Pareto Genetic Algorithm (NPGA). The main advantages of the proposed approach is that it does not require the definition of any extra parameters, other than those required by an evolution strategy. The performance of the proposed approach is shown to be highly competitive with respect to other constraint-handling techniques representative of the state-of-the-art in the area when using a set of well-known benchmarks.
\end{abstract}

\section{Introduction}

Evolutionary Algorithms (EAs) are heuristic methods that have been successfully applied to a wide set of application domains [10], both, in global (single-objective) and in multiobjective optimization. Nevertheless, EAs are an unconstrained search technique and lack an explicit mechanism to deal with constrained search spaces. This has motivated the development of a considerable number of approaches to incorporate constraints into the fitness function of an EA $[11,1]$.

The most common approach adopted to deal with constrained search spaces is the use of penalty functions [12]. When using a penalty function, the amount of constraint violation is used to punish or "penalize" an infeasible solution so that feasible solutions are favored by the selection process. Despite the popularity of penalty functions, they have several drawbacks from which the main one is that they require a careful fine tuning of the penalty factors that accurately estimates the degree of penalization to be applied so that we can approach efficiently the feasible region $[15,1]$.

Evolution Strategies (ES) have been found not only efficient in solving a wide variety of optimization problems [4], but also have a strong theoretical background [14]. Motivated by the fact that some of the most recent (and most competitive) approaches to incorporate constraints into an EA use an ES (see for example [13, 5]), Mezura \& Coello [9] proposed a Simple Evolution Strategy (SES) to solve constrained problems. This approach is based on a double mechanism: (1) the original selfadaptation mechanism of the ES to sample the search space and (2) a selection mechanism that chooses solutions based on feasibility as new starting points for the search. However, our SES presented some drawbacks that are improved in the current work. Our main improvement consists of a diversity mechanism that allows the best of the $\lambda$ individuals (based on feasibility and objective function value) in the current generation (and not only the child formed by all these individuals) to replace the current solution. We also allow the individual (from the $\lambda$ created) with the best objective function value to replace the current solution regardless of its feasibility. This mechamism is controlled by a stochastic parameter called Selection Ration $\left(S_{r}\right)$. This parameter indicates the number of times (as a percentage) that normal selection (i.e., deterministic between the current solution and the child formed by all the $\lambda$ candidate solutions) will take place. The remaining $1-S_{r}$ times, either the best solution (from the $\lambda$ ) based on feasibility and fitness or the individual (also from the $\lambda$ ) with the best value of the objective function (regardless of feasibility) will replace the current solution. In the current work, equality constraints are transformed into inequality constraints using a dynamic parameterless tolerance. In the remainder of the paper we will call "candidate solution" to those $\lambda$ individuals created on each generation and we will call "child" to the solution created from the combination of the $\lambda$ solutions, the current solution is the start point of each generation.

This paper is organized as follows: in Section 2 a short survey of constraint-handling techniques similar to our own is presented. Section 3 provides a detailed description of the proposed approach. Section 4, describes the test functions used and presents the results obtained. Section 5 provides a discussion of our results. Finally, Section 6 provides our conclusions and some possible paths for future research.

\section{Previous Work}

The use of tournament selection based on feasibility rules has been explored by other authors. Jiménez and Verdegay [7] proposed an approach similar to a min-max formulation used in multiobjective optimization combined with tournament selection. The rules used by them are similar to those adopted in this work. However, Jiménez and Verdegay's approach lacks an explicit mechanism to avoid the premature convergence produced by the random sampling of the feasible region because their approach is guided by the first feasible solution found. Deb [3] used the same tournament rules previously indicated in his approach. However, Deb proposed to use niching as a diversity mechanism, which introduces some extra computational time (niching 
has time-complexity $O\left(N^{2}\right)$ ). In Deb's approach, feasible solutions are always considered better than infeasible ones. This contradicts the idea of allowing infeasible individuals to remain in the population. Therefore, this approach will have difficulties in problems in which the global optimum lies on the boundary between the feasible and the infeasible regions. Coello \& Mezura [2] used tournament selection based on feasibility rules. They also adopted nondominance checkings using a sample of the population (as the multiobjective optimization approach called NPGA [6]). They adopted a user-defined parameter $S_{r}$, to control the diversity in the population. This approach provided good results in some well-known engineering problems and in some benchmark problems, but presented problems when facing high dimensionality [2].

The three approaches discussed before are based on a genetic algorithm. However, several of the most competitive constraint-handling approaches known to date are based on an ES (e.g., Stochastic Ranking [13] and the Adaptive Segregated Constraint Handling Evolutionary Algorithm (ASCHEA) [5]).

\section{Description of the Approach}

The motivation of this work was twofold:

1. We hypothesized that the use of an ES for constrained optimization would be beneficial (compared to the use of a genetic algorithm).

2. We were also aware that having a good mechanism to maintain diversity is of great importance to produce a constraint-handling approach that is competitive with the techniques representative of the state-of-the-art in the area.

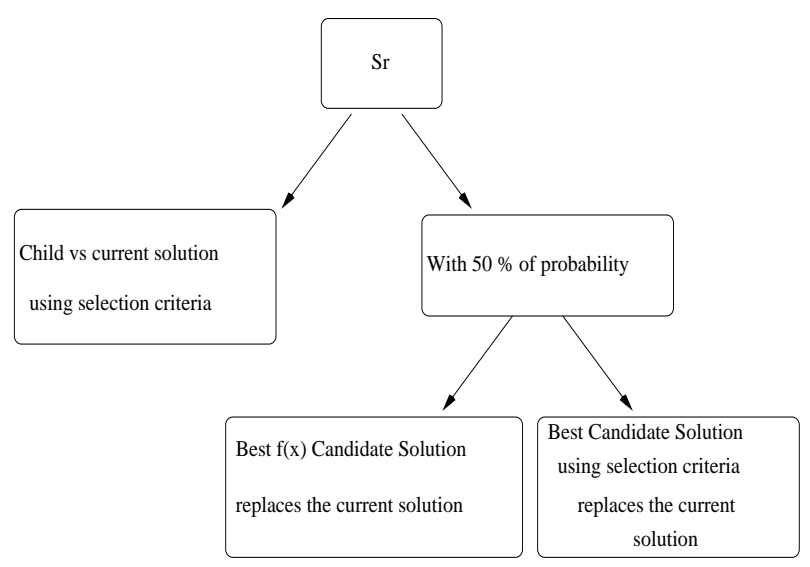

Figure 1: Diagram that illustrates the diversity mechanism implemented for our SES.

In [9], we introduced the so-called Simple Evolution Strategy (SES), which is based on two mechanisms:

- The self-adaptation mechanism of an ES, which helps our approach to sample the search space well enough as to reach the feasible region reasonably fast.
- The use of tournaments based on feasibility, which are adopted to guide the search in such a way that the global optimum can be approached efficiently.

The three simple selection criteria used in our tournaments are the following (binary tournaments are adopted):

- Between 2 feasible solutions, the one with the highest fitness value wins (assuming a maximization problem/task).

- If one solution is feasible and the other one is infeasible, the feasible solution wins.

- If both solutions are infeasible, the one with the lowest sum of constraint violation is preferred.

Our SES uses the $1 / 5$-success rule for auto-adapting the $\sigma$ value of our ES. By using just one $\sigma$ value and one fitness function evaluation per generation, the resulting computational cost (per generation) of our approach is very low.

This first version of our approach provided very competitive results [9]. However, it presented premature convergence in some problems due to the high selection pressure caused by the tournaments performed. This motivated the changes introduced in this paper.

The new version of our algorithm has a process controlled by a parameter (that is not defined by the user) called Selection Ratio $\left(S_{r}\right)$. This parameter was introduced in [2] and it refers to the percentage of selections that will be performed in a deterministic way (as used in the original version of our SES where the child replaces the current solution based on the three selection criteria). In the remaining $1-S_{r}$ selections, there are two choices: (1) either the candidate solution with the best value of the objective function will replace the current solution (regardless of its feasibility) or (2) the best candidate solution (based on the three selection criteria) will replace the current solution. Both options are given a $50 \%$ probability each (see Figure 1).

The $S_{r}$ parameter is adapted online using the fitness value of the current solution during an interval of time (number of generations). The "mean deviation" $\left(M_{d}\right)$ of the current solution over a certain number of generations is calculated in order to know how different has been the current solution. All the fitnesses are normalized in order to obtain a value between 0 and 1 . The expression used to adapt the $S_{r}$ value is the following:

$$
S_{r}(t)= \begin{cases}S_{r}(t-\text { interval }) / 1.001 & \text { if } M_{d}<0.1 \\ S_{r}(t-\text { interval }) * 1.001 & \text { if } M_{d}>0.2 \\ S_{r}(t-\text { interval }) & \text { if } 0.1 \leq M_{d} \leq 0.2\end{cases}
$$

where interval is defined as a percentage of the maximum number of generations. For example if the interval is defined as 0.05 and the number of generations is 100 , the update process will take place at every 5 generations. As can be seen, $S_{r}$ will be decreased if the current solution has not significantly changed during the given interval (i.e., $M_{d}<0.1$ ) allowing a candidate solution (which may be infeasible) with a good fitness value to replace the current solution. This is meant to increase diversity. On the other hand, $S_{r}$ will increase if the solution has been significantly different (i.e., $M_{d}>0.2$ ) during the interval, thus favoring 


\begin{tabular}{|c|c|c|c|c|c|c|}
\hline \multirow[b]{2}{*}{ Problem } & \multicolumn{6}{|c|}{ SES } \\
\hline & Optimal & Best & Mean & Median & Worst & St. Dev. \\
\hline TF1 & -15 & -15.000000 & -15.000000 & -15.000000 & -15.000000 & 0.000000 \\
\hline TF2 & 0.803619 & 0.803569 & 0.769612 & 0.782466 & 0.702322 & 0.027547 \\
\hline TF3 & 1 & 1.004329 & 1.003563 & 1.003669 & 1.002604 & 0.000423 \\
\hline TF4 & -30665.539 & -30665.539062 & -30665.539062 & -30665.539062 & -30665.539062 & 0.000000 \\
\hline TF5 & -6961.814 & -6961.813965 & -6961.813965 & -6961.813965 & -6961.813965 & 0.000000 \\
\hline TF6 & 24.306 & 24.313972 & 24.418837 & 24.419113 & 24.560797 & 0.071159 \\
\hline TF7 & 0.095825 & 0.095826 & 0.095784 & 0.095826 & 0.095473 & 0.000104 \\
\hline TF8 & 680.63 & 680.669189 & 680.809829 & 680.797882 & 681.199646 & 0.122624 \\
\hline TF9 & 7049.25 & 7057.044434 & 10771.416895 & 10935.448730 & 16375.266602 & 2524.075298 \\
\hline TF10 & 0.75 & 0.749018 & 0.749179 & 0.749062 & 0.750647 & 0.000316 \\
\hline TF11 & 1 & 1.000000 & 1.000000 & 1.000000 & 1.000000 & 0.000000 \\
\hline TF12 & 0.053950 & 0.053964 & 0.264135 & 0.438692 & 0.544346 & 0.206214 \\
\hline
\end{tabular}

Table 1: Statistical results obtained of the 30 runs performed using the new SES with the 12 test problems adopted.

deterministic selection to impel convergence. $S_{r}$ will keep its current value if the variation of the current solution in the interval has been moderated (i.e., $0.1 \leq M_{d} \leq 0.2$ ).

In order to always keep the best solution found during the process, a superelitist mechanism is included. Its only goal is to keep the best feasible solution found. This is required because the diversity mechanism adopted makes the current solution to be replaced by another solution which is not necessarily better and may be infeasible. Its implementation does not add any significant extra computational or storage cost to the algorithm.

To deal with equality constraints, a parameterless dynamic mechanism similar to that used in ASCHEA [5] is adopted. The tolerance value $\epsilon$ is decreased with respect to the current generation using the following expression:

$$
\epsilon_{j}(t+1)=\epsilon_{j}(t) / 1.000001
$$

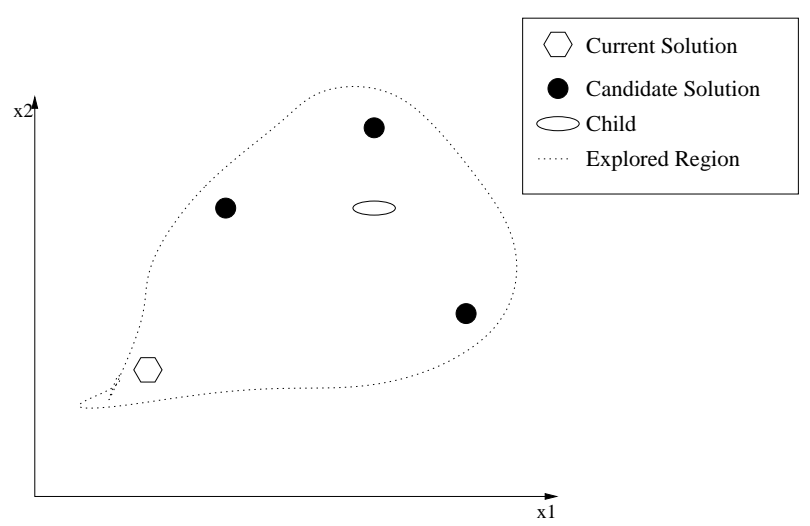

Figure 2: Diagram that illustrates the explored region of the search space in the new version of our SES. In the old version only the points in white (child and current solution) could be selected.

It is worth emphasizing that allowing a candidate solution to replace the current solution in a $(1+\lambda)$-ES (used by our SES) enhances the search power (global and local) of the ES because it explores more deeply the regions that surround the current solution. As the $\sigma$ value is high early in the process, it will generate candidate solutions on far regions of the search space (global search). This will allow to sample the search space in a better way as to select the most promising point. The same behavior will occur later in the process, but in this case the points close to the current solution will be the more deeply explored (local search). A graphical illustration of this behavior is provided in Figure 2.

Another aspect to note is that in order to maintain the low computational cost of our SES, the candidate solutions are only evaluated if some of them are to replace the current solution. In the same way, the child will be evaluated only if it is to compete against the current solution. The pseudocode of this approach is shown in Figure 3.

\section{Experiments and Result}

To evaluate the performance of the new approach we used 12 of the test functions described in [13]. The test functions chosen contain characteristics that are representative of what can be considered "difficult" global optimization problems for an EA. Their expressions are provided next.

- TF1:

Minimize: $f(\vec{x})=5 \sum_{i=1}^{4} x_{i}-5 \sum_{i=1}^{4} x_{i}^{2}-\sum_{i=5}^{13} x_{i}$ subject to:

$$
\begin{aligned}
& g_{1}(\vec{x})=2 x_{1}+2 x_{2}+x_{10}+x_{11}-10 \leq 0 \\
& g_{2}(\vec{x})=2 x_{1}+2 x_{3}+x_{10}+x_{12}-10 \leq 0 \\
& g_{3}(\vec{x})=2 x_{2}+2 x_{3}+x_{11}+x_{12}-10 \leq 0 \\
& g_{4}(\vec{x})=-8 x_{1}+x_{10} \leq 0 \\
& g_{5}(\vec{x})=-8 x_{2}+x_{11} \leq 0 \\
& g_{6}(\vec{x})=-8 x_{3}+x_{12} \leq 0 \\
& g_{7}(\vec{x})=-2 x_{4}-x_{5}+x_{10} \leq 0 \\
& g_{8}(\vec{x})=-2 x_{6}-x_{7}+x_{11} \leq 0 \\
& g_{9}(\vec{x})=-2 x_{8}-x_{9}+x_{12} \leq 0
\end{aligned}
$$

where the bounds are $0 \leq x_{i} \leq 1(i=1, \ldots, 9)$, $0 \leq x_{i} \leq 100(i=10,11,12)$ and $0 \leq x_{13} \leq 1$. The global optimum is at $x^{*}=(1,1,1,1,1,1,1,1,1,3,3,3,1)$ where $f\left(x^{*}\right)=-15$. Constraints $g_{1}, g_{2}, g_{3}, g_{4}, g_{5}$ and $g_{6}$ are active.

\section{- TF2:}

Maximize: $f(\vec{x})=\left|\frac{\sum_{i=1}^{n} \cos ^{4}\left(x_{i}\right)-2 \prod_{i=1}^{n} \cos ^{2}\left(x_{i}\right)}{\sqrt{\sum_{i=1}^{n} i x_{i}^{2}}}\right|$ 
subject to:

$$
\begin{aligned}
g_{1}(\vec{x}) & =0.75-\prod_{i=1}^{n} x_{i} \leq 0 \\
g_{2}(\vec{x}) & =\sum_{i=1}^{n} x_{i}-7.5 n \leq 0
\end{aligned}
$$

where $n=20$ and $0 \leq x_{i} \leq 10(i=1, \ldots, n)$. The global maximum is unknown; the best reported solution is [13] $f\left(x^{*}\right)=0.803619$. Constraint $g_{1}$ is close to being active $\left(g_{1}=-10^{-8}\right)$.

- TF3:

Maximize: $f(\vec{x})=(\sqrt{n})^{n} \prod_{i=1}^{n} x_{i}$

subject to:

$h(\vec{x})=\sum_{i=1}^{n} x_{i}^{2}-1=0$

where $n=10$ and $0 \leq x_{i} \leq 1(i=1, \ldots, n)$. The global maximum is at $x_{i}^{*}=1 / \sqrt{n}(i=1, \ldots, n)$ where $f\left(x^{*}\right)=1$.

- TF4:

Minimize: $f(\vec{x})=5.3578547 x_{3}^{2}+0.8356891 x_{1} x_{5}+$ $37.293239 x_{1}-40792.141$

subject to:

$g_{1}(\vec{x})=85.334407+0.0056858 x_{2} x_{5}+$ $0.0006262 x_{1} x_{4}-0.0022053 x_{3} x_{5}-92 \leq 0$

$g_{2}(\vec{x})=-85.334407-0.0056858 x_{2} x_{5}-$ $0.0006262 x_{1} x_{4}+0.0022053 x_{3} x_{5} \leq 0$

$g_{3}(\vec{x})=80.51249+0.0071317 x_{2} x_{5}+$ $0.0029955 x_{1} x_{2}+0.0021813 x_{3}^{2}-110 \leq 0$

$g_{4}(\vec{x})=-80.51249-0.0071317 x_{2} x_{5}-$ $0.0029955 x_{1} x_{2}-0.0021813 x_{3}^{2}+90 \leq 0$

$g_{5}(\vec{x})=9.300961+0.0047026 x_{3} x_{5}+$ $0.0012547 x_{1} x_{3}+0.0019085 x_{3} x_{4}-25 \leq 0$

$g_{6}(\vec{x})=-9.300961-0.0047026 x_{3} x_{5}-$

$0.0012547 x_{1} x_{3}-0.0019085 x_{3} x_{4}+20 \leq 0$

where: $78 \leq x_{1} \leq 102,33 \leq x_{2} \leq 45,27 \leq$ $x_{i} \leq 45(i=3,4,5)$. The optimum solution is $x^{*}=$ $(78,33,29.995256025682,45,36.775812905788)$ where $f\left(x^{*}\right)=-30665.539$. Constraints $g_{1}$ y $g_{6}$ are active.

\section{- TF5}

Minimize: $f(\vec{x})=\left(x_{1}-10\right)^{3}+\left(x_{2}-20\right)^{3}$ subject to:

$g_{1}(\vec{x})=-\left(x_{1}-5\right)^{2}-\left(x_{2}-5\right)^{2}+100 \leq 0$

$g_{2}(\vec{x})=\left(x_{1}-6\right)^{2}+\left(x_{2}-5\right)^{2}-82.81 \leq 0$

where $13 \leq x_{1} \leq 100$ and $0 \leq x_{2} \leq 100$. The optimum solution is $x^{*}=(14.095,0.84296)$ where $f\left(x^{*}\right)=-6961.81388$. Both constraints are active.

\section{- TF6}

Minimize: $f(\vec{x})=x_{1}^{2}+x_{2}^{2}+x_{1} x_{2}-14 x_{1}-16 x_{2}+$ $\left(x_{3}-10\right)^{2}+4\left(x_{4}-5\right)^{2}+\left(x_{5}-3\right)^{2}+2\left(x_{6}-1\right)^{2}+$ $5 x_{7}^{2}+7\left(x_{8}-11\right)^{2}+2\left(x_{9}-10\right)^{2}+\left(x_{10}-7\right)^{2}+45$ subject to:

$g_{1}(\vec{x})=-105+4 x_{1}+5 x_{2}-3 x_{7}+9 x_{8} \leq 0$

$g_{2}(\vec{x})=10 x_{1}-8 x_{2}-17 x_{7}+2 x_{8} \leq 0$

$g_{3}(\vec{x})=-8 x_{1}+2 x_{2}+5 x_{9}-2 x_{10}-12 \leq 0$

$g_{4}(\vec{x})=3\left(x_{1}-2\right)^{2}+4\left(x_{2}-3\right)^{2}+2 x_{3}^{2}-7 x_{4}-120 \leq 0$

$g_{5}(\vec{x})=5 x_{1}^{2}+8 x_{2}+\left(x_{3}-6\right)^{2}-2 x_{4}-40 \leq 0$

$g_{6}(\vec{x})=x_{1}^{2}+2\left(x_{2}-2\right)^{2}-2 x_{1} x_{2}+14 x_{5}-6 x_{6} \leq 0$

$g_{7}(\vec{x})=0.5\left(x_{1}-8\right)^{2}+2\left(x_{2}-4\right)^{2}+3 x_{5}^{2}-x_{6}-30 \leq 0$

$g_{8}(\vec{x})=-3 x_{1}+6 x_{2}+12\left(x_{9}-8\right)^{2}-7 x_{10} \leq 0$

where $-10 \leq x_{i} \leq 10(i=1, \ldots, 10)$. The global optimum is $x^{*}=(2.171996,2.363683,8.773926$, $5.095984,0.9906548,1.430574,1.321644$, $9.828726,8.280092,8.375927)$ where $f\left(x^{*}\right)=$ 24.3062091. Constraints $g_{1}, g_{2}, g_{3}, g_{4}, g_{5}$ and $g_{6}$ are active.

- TF7

Maximize: $f(\vec{x})=\frac{\sin ^{3}\left(2 \pi x_{1}\right) \sin \left(2 \pi x_{2}\right)}{x_{1}^{3}\left(x_{1}+x_{2}\right)}$

subject to:

$g_{1}(\vec{x})=x_{1}^{2}-x_{2}+1 \leq 0$

$g_{2}(\vec{x})=1-x_{1}+\left(x_{2}-4\right)^{2} \leq 0$

where $0 \leq x_{1} \leq 10$ and $0 \leq x_{2} \leq 10$. The optimum solution is located at $x^{*}=(1.2279713,4.2453733)$ where $f\left(x^{*}\right)=0.095825$. The solutions is located within the feasible region.

\section{- TF8}

Minimize: $f(\vec{x})=\left(x_{1}-10\right)^{2}+5\left(x_{2}-12\right)^{2}+x_{3}^{4}+$ $3\left(x_{4}-11\right)^{2}+10 x_{5}^{6}+7 x_{6}^{2}+x_{7}^{4}-4 x_{6} x_{7}-10 x_{6}-8 x_{7}$ subject to:

$g_{1}(\vec{x})=-127+2 x_{1}^{2}+3 x_{2}^{4}+x_{3}+4 x_{4}^{2}+5 x_{5} \leq 0$

$g_{2}(\vec{x})=-282+7 x_{1}+3 x_{2}+10 x_{3}^{2}+x_{4}-x_{5} \leq 0$

$g_{3}(\vec{x})=-196+23 x_{1}+x_{2}^{2}+6 x_{6}^{2}-8 x_{7} \leq 0$

$g_{4}(\vec{x})=4 x_{1}^{2}+x_{2}^{2}-3 x_{1} x_{2}+2 x_{3}^{2}+5 x_{6}-11 x_{7} \leq 0$

where $-10 \leq x_{i} \leq 10 \quad(i=$ $1, \ldots, 7)$. The global optimum is $x^{*}=$ $(2.330499,1.951372,-0.4775414,4.365726$,

$-0.6244870,1.038131,1.594227) \quad$ where $f\left(x^{*}\right)=680.6300573$. Two constraints are active $\left(g_{1}\right.$ and $\left.g_{4}\right)$.

\section{- TF9}

Minimize: $f(\vec{x})=x_{1}+x_{2}+x_{3}$

subject to:

$g_{1}(\vec{x})=-1+0.0025\left(x_{4}+x_{6}\right) \leq 0$

$g_{2}(\vec{x})=-1+0.0025\left(x_{5}+x_{7}-x_{4}\right) \leq 0$

$g_{3}(\vec{x})=-1+0.01\left(x_{8}-x_{5}\right) \leq 0$

$g_{4}(\vec{x})=-x_{1} x_{6}+833.33252 x_{4}+100 x_{1}-$ $83333.333 \leq 0$

$g_{5}(\vec{x})=-x_{2} x_{7}+1250 x_{5}+x_{2} x_{4}-1250 x_{4} \leq 0$

$g_{6}(\vec{x})=-x_{3} x_{8}+1250000+x_{3} x_{5}-2500 x_{5} \leq 0$

where $100 \leq x_{1} \leq 10000,1000 \leq x_{i} \leq 10000$, $(i=2,3), 10 \leq x_{i} \leq 1000,(i=4, \ldots, 8)$. The global optimum is: $x^{*}=(579.19,1360.13,5109.92$, 182.01, 295.5985, 217.9799, 286.40, 395.5979), where $f\left(x^{*}\right)=7049.25 . g_{1}, g_{2}$ and $g_{3}$ are active. 


\begin{tabular}{|c|c|c|c|c|c|c|c|}
\hline Problem & n & Function type & $\rho$ & LI & NI & LE & NE \\
\hline \hline TF1 & 13 & quadratic & $0.0003 \%$ & 9 & 0 & 0 & 0 \\
\hline TF2 & 20 & nonlinear & $99.9973 \%$ & 2 & 0 & 0 & 0 \\
\hline TF3 & 10 & nonlinear & $0.0026 \%$ & 0 & 0 & 0 & 1 \\
\hline TF4 & 5 & quadratic & $27.0079 \%$ & 4 & 2 & 0 & 0 \\
\hline TF5 & 2 & nonlinear & $0.0057 \%$ & 0 & 2 & 0 & 0 \\
\hline TF6 & 10 & quadratic & $0.0000 \%$ & 3 & 5 & 0 & 0 \\
\hline TF7 & 2 & nonlinear & $0.8581 \%$ & 0 & 2 & 0 & 0 \\
\hline TF8 & 7 & nonlinear & $0.5199 \%$ & 0 & 4 & 0 & 0 \\
\hline TF9 & 8 & linear & $0.0020 \%$ & 6 & 0 & 0 & 0 \\
\hline TF10 & 2 & quadratic & $0.0973 \%$ & 0 & 0 & 0 & 1 \\
\hline TF11 & 3 & quadratic & $4.7697 \%$ & 0 & $9^{3}$ & 0 & 0 \\
\hline TF12 & 5 & nonlinear & $0.0000 \%$ & 0 & 0 & 1 & 2 \\
\hline
\end{tabular}

Table 3: Values of $\rho$ for the 12 test functions chosen.

\section{- TF10}

Minimize: $f(\vec{x})=x_{1}^{2}+\left(x_{2}-1\right)^{2}$

subject to:

$h(\vec{x})=x_{2}-x_{1}^{2}=0$

where: $-1 \leq x_{1} \leq 1,-1 \leq x_{2} \leq 1$. The optimum solution is $x^{*}=( \pm 1 / \sqrt{2}, 1 / 2)$ where $f\left(x^{*}\right)=0.75$.

- TF11

Maximize: $f(\vec{x})=\frac{100-\left(x_{1}-5\right)^{2}-\left(x_{2}-5\right)^{2}-\left(x_{3}-5\right)^{2}}{100}$ subject to:

$g_{1}(\vec{x})=\left(x_{1}-p\right)^{2}+\left(x_{2}-q\right)^{2}+\left(x_{3}-r\right)^{2}-0.0625 \leq 0$

where $0 \leq x_{i} \leq 10(i=1,2,3)$ and $p, q, r=1,2, \ldots, 9$. The feasible region of the search space consists of $9^{3}$ disjointed spheres. A point $\left(x_{1}, x_{2}, x_{3}\right)$ is feasible if and only if there exist $p, q, r$ such the above inequality (4) holds. The global optimum is located at $x^{*}=(5,5,5)$ where $f\left(x^{*}\right)=1$.

- TF12

Minimize: $f(\vec{x})=e^{x_{1} x_{2} x_{3} x_{4} x_{5}}$

subject to:

$g_{1}(\vec{x})=x_{1}^{2}+x_{2}^{2}+x_{3}^{2}+x_{4}^{2}+x_{5}^{2}-10=0$

$g_{2}(\vec{x})=x_{2} x_{3}-5 x_{4} x_{5}=0$

$g_{3}(\vec{x})=x_{1}^{3}+x_{2}^{3}+1=0$

where $-2.3 \leq x_{i} \leq 2.3(i=1,2)$ and $-3.2 \leq$ $x_{i} \leq 3.2(i=3,4,5)$. The optimum solution is $x^{*}=$ $(-1.717143,1.595709,1.827247,-0.7636413$,

$-0.763645)$ where $f\left(x^{*}\right)=0.0539498$.

To get a measure of the difficulty of solving each of these problems, a $\rho$ metric (as suggested by Koziel and Michalewicz [8]) was computed using the following expression:

$$
\rho=|F| /|S|
$$

where $|F|$ is the number of feasible solutions and $|S|$ is the total number of solutions randomly generated. In this work, $S=1,000,000$ random solutions.

The different values of $\rho$ for each of the functions chosen are shown in Table 3, where $n$ is the number of decision variables, LI is the number of linear inequalities, NI the number of nonlinear inequalities, LE is the number of linear equalities and NE is the number of nonlinear equalities.
The parameters used in the experiments are the following (30 runs were performed for each problem): the total number of fitness function evaluations was set to 330,000 . Equality constraints were transformed into inequalities using an initial tolerance value of 0.001 . The initial values for the $(1+\lambda)$-ES parameters were: $\sigma=4.0, C=0.99$, $\lambda=3$, and maximum number of generations $=275,000$. The interval of the $S_{r}$ updates was almost negligible (0.9). This means that the update will take place until generation 247,500 . We anticipated that our approach would not be too sensitive to the $S_{r}$ parameter and our experiments confirmed this hypothesis. The statistical results are presented in Table 1. For problems TF3, TF4, TF7 and TF10 the results seem to improve the corresponding optimum. It is due to the fact that either we use a small tolerance for equality constraints (TF3 and TF10), or we round the results (TF4 and TF7).

\section{Discussion of Results}

In Tables 2, 4, 5 and 6, we compare the new SES against the last (old) SES, the Homomorphous Maps [8], Stochastic Ranking [13] and ASCHEA [5], respectively. Such approaches were selected for comparison because they are representative of the state-of-the-art in the area. There are several issues derived from this comparison that deserve some discussion:

- The new SES was able to converge to the global optimum in 7 of the test 12 functions used (TF1, TF3, TF4, TF5, TF7, TF10 and TF11), and it was able to converge very close to the optimum in TF2, TF6, TF8, TF9 and TF12.

- With respect to the last version of the SES (Table 2), this New SES improved the robustness of the results in problems TF1, TF2, TF4, TF6 and TF10. Also, the quality of the results was improved in problems TF9 and TF12.

- With respect to the Homomorphous Maps (see Table 4), we can see that our SES converged to a better "best" solution in 9 problems (TF1, TF2, TF3, TF4, TF5, TF6, TF8, TF9 and TF11). Also, it found a better average and a better "worst" solution in 8 problems (TF1, TF3, TF4, TF5, TF6, TF7, TF8, and TF11). Thus, it should be clear that our SES had a highly competitive performance, even improving the results of the Homomorphous Maps in several test functions. No comparison was made with problem TF12 because such results were not available.

- With respect to Stochastic Ranking (see Table 5), the new SES was able to converge to similar "best" solutions in 10 problems (TF1, TF3, TF4, TF5, TF6,TF7, TF8, TF10, TF11 and TF12). SES found a slightly better result in problem TF2. Moreover, SES found a similar average solution in 8 problems (TF1, TF3, TF4, TF6, TF7,TF8, TF10, and TF11). SES found a better average solution in problem TF5. Finally, SES found similar "worst" solutions in 5 problems (TF1, TF3, TF4, TF6 and TF11) 


\begin{tabular}{|c|c|c|c|c|c|c|c|}
\hline & & \multicolumn{2}{|c|}{ Best Result } & \multicolumn{2}{|c|}{ Mean Result } & \multicolumn{2}{|c|}{ Worst Result } \\
\hline $\mathbf{P}$ & Optimal & $\overline{\text { NEW }}$ & OLD & $\overline{\text { NEW }}$ & OLD & $\overline{\text { NEW }}$ & OLD \\
\hline TF1 & -15 & -15.000000 & -15.000000 & -15.000000 & -14.848614 & -15.000000 & -12.999997 \\
\hline TF2 & 0.803619 & 0.803569 & 0.793083 & 0.769612 & 0.698932 & 0.702322 & 0.576079 \\
\hline TF3 & 1 & 1.004329 & 1.000497 & 1.003563 & 1.000491 & 1.002604 & 1.000424 \\
\hline TF4 & -30665.539 & -30665.539062 & -30665.539062 & -30665.539062 & -30665.539062 & -30665.539062 & -30663.496094 \\
\hline TF5 & -6961.814 & -6961.813965 & -6961.813965 & -6961.813965 & -6961.813965 & -6961.813965 & -6961.813965 \\
\hline TF6 & 24.306 & 24.313972 & 24.368050 & 24.418837 & 24.702525 & 24.560797 & 25.516653 \\
\hline TF7 & 0.095825 & 0.095826 & 0.095826 & 0.095784 & 0.095826 & 0.095473 & 0.095826 \\
\hline TF8 & 680.63 & 680.669189 & 680.631653 & 680.809829 & 680.673645 & 681.199646 & 680.915100 \\
\hline TF9 & 7049.25 & 7057.044434 & - & 10771.416895 & - & 16375.266602 & - \\
\hline TF10 & 0.75 & 0.749018 & 0.749900 & 0.749179 & 0.784395 & 0.750647 & 0.879522 \\
\hline TF11 & 1 & 1.000000 & 1.000000 & 1.000000 & 1.000000 & 1.000000 & 1.000000 \\
\hline TF12 & 0.053950 & 0.053964 & - & 0.264135 & - & 0.544346 & - \\
\hline
\end{tabular}

Table 2: Comparison of results between the new SES and the old SES proposed in [9]. "-" means no feasible solutions were found.

\begin{tabular}{|c|c|c|c|c|c|c|c|}
\hline & & \multicolumn{2}{|c|}{ Best Result } & \multicolumn{2}{|c|}{ Mean Result } & \multicolumn{2}{|c|}{ Worst Result } \\
\hline Problem & Optimal & SES & HM & SES & $\overline{\mathbf{H M}}$ & SES & $\overline{\mathrm{HM}}$ \\
\hline TF1 & -15 & -15.000000 & -14.7886 & -15.000000 & -14.7082 & -15.000000 & -14.6154 \\
\hline TF2 & 0.803619 & 0.803569 & 0.79953 & 0.769612 & 0.79671 & 0.702322 & 0.79119 \\
\hline TF3 & 1 & 1.004329 & 0.9997 & 1.003563 & 0.9989 & 1.002604 & 0.9978 \\
\hline TF4 & -30665.539 & -30665.539062 & -30664.5 & -30665.539062 & -30655.3 & -30665.539062 & -30645.9 \\
\hline TF5 & -6961.814 & -6961.813965 & -6952.1 & -6961.813965 & -6342.6 & -6961.813965 & -5473.9 \\
\hline TF6 & 24.306 & 24.313972 & 24.620 & 24.418837 & 24.826 & 24.560797 & 25.069 \\
\hline TF7 & 0.095825 & 0.095826 & 0.0958250 & 0.095784 & 0.0891568 & 0.095473 & 0.0291438 \\
\hline TF8 & 680.63 & 680.669189 & 680.91 & 680.809829 & 681.16 & 681.199646 & 683.18 \\
\hline TF9 & 7049.25 & 7057.044434 & 7147.9 & 10771.416895 & 8163.6 & 16375.266602 & 9659.3 \\
\hline TF10 & 0.75 & 0.749018 & 0.75 & 0.749179 & 0.75 & 0.750647 & 0.75 \\
\hline TF11 & 1 & 1.000000 & 0.999999857 & 1.000000 & 0.999134613 & 1.000000 & 0.991950498 \\
\hline TF12 & 0.053950 & 0.053964 & $N A$ & 0.264135 & $N A$ & 0.544346 & $N A$ \\
\hline
\end{tabular}

Table 4: Comparison of results between our approach (SES) and the Homomorphous Maps (HM) [8] NA = Not Available.

and reached a better "worst" individual in problem TF5. Though the Stochastic Ranking performed with a little more consistency, SES performed almost at a similar level than this highly competitive approach.

- Finally, with respect to ASCHEA (see Table 6), the new SES converged to similar "best" results in 7 problems (TF1, TF3, TF4, TF5, TF7, TF8 and TF10) and it found better "best"results in 3 problems (TF2, TF6 and TF9). A better average result was found by the SES in 4 problems (TF1, TF2, TF3 and TF6), and a similar average solution was reached in 4 problems (TF4, TF5, TF8 and TF10). Note that we didn't present any results for ASCHEA in problems TF11 and TF12 because such results were not available.

From the previous comparison, we can see that the new SES produced very competitive results with respect to three techniques representative of the state-of-the-art in constrained optimization. The new SES can deal with highly constrained problems, problems with low (TF5 and TF7) and high (TF1, TF2, TF3, TF6) dimensionality, with different types of combined constraints (linear, nonlinear, equality and inequality) and with very large (TF2) or very small (TF12) or even disjoint (TF11) feasible regions. However, our approach presented some robustness problems in TF9. This function has a very large search space (because of the intervals of the decision variables). Therefore, our SES required to explore several regions of the search space in order to find promising areas consistently. This can be obtained more easily by using a population-based ES (e.g., a $(\mu+\lambda)$ $\mathrm{ES})$.

Besides still being a very simple approach, it is worth reminding that SES does not require any extra parameters (besides those used with an ES) because the $S_{r}$ is adapted online. In contrast, the Homomorphous Maps require an additional parameter (called $v$ ) which has to be found empirically [8]. Stochastic ranking requires the definition of a parameter called $P_{f}$, whose value has an important impact on the performance of the approach [13]. ASCHEA also requires the definition of several extra parameters, and in its latest version, it uses niching, which is a process that also has at least one additional parameter [5].

Measuring the computational cost, the number of fitness function evaluations (FFE) performed by our approach is lower than the other techniques with respect to which it was compared. Our approach performed 330,000 FFE. Stochastic ranking performed 350,000 FFE, the Homomorphous Maps performed 1,400,000 FFE, and ASCHEA performed 1,500,000 FFE.

It is interesting to note that with a very small population size (only 3 individuals), the search power is improved when we allow to the candidate solutions to replace (in a deterministic way) the current solution regardless of their feasibility. 


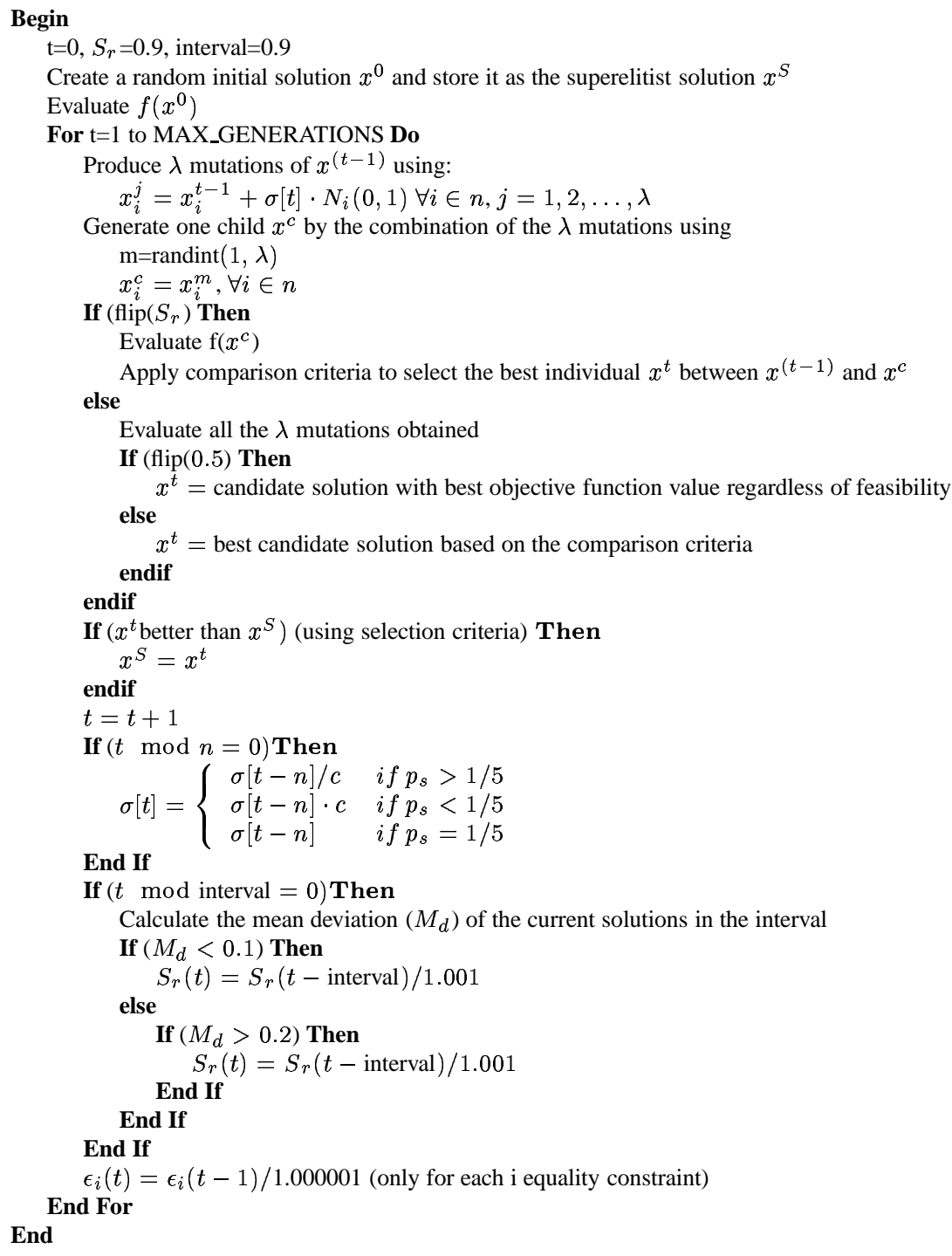

Figure 3: Our algorithm of SES ( $n$ is the number of decision variables of the problem, $f l i p(P)$ is a function that returns TRUE with probability $P$ ).

\section{Conclusions and Future Work}

We have introduced the addition of a diversity maintenance mechanism to a simple ES previously proposed to solve constrained optimization problems [9]. Such a mechanism was found to improve the quality and robustness of our approach.

We also introduced the use of an online adaptive mechanism that automatically updates the value of the $S_{r}$ parameter (responsible for controlling diversity in the population). As its predecessor, the new SES does not require a penalty function or any extra parameters (other than the original parameters of an ES) to bias the search towards the feasible region of a problem. Additionally, this improved approach has a low computational cost and it is easy to implement.

Our main path for future research is to explore in more detail the impact of the $S_{r}$ parameter on the performance of the approach. We also intend to use our SES to solve some engineering (real-world) optimization problems.

\section{Acknowledgments}

The first author acknowledges support from the mexican Consejo Nacional de Ciencia y Tecnología (CONA$\mathrm{CyT}$ ) through a scholarship to pursue graduate studies at CINVESTAV-IPN's Electrical Engineering Department. The second author acknowledges support from CONACyT through project number 32999-A.

\section{Bibliography}

[1] C. A. Coello Coello. Theoretical and Numerical Constraint Handling Techniques used with Evolutionary Algorithms: A Survey of the State of the Art. Computer Methods in Applied Mechanics and Engineering, 191(11-12):1245-1287, January 2002.

[2] C. A. Coello Coello and E. Mezura-Montes. Handling Constraints in Genetic Algorithms Using Dominance-Based Tournaments. In I. Parmee, editor, Proceedings of the Fifth International Conference on Adaptive Computing De- 


\begin{tabular}{|c|c|c|c|c|c|c|c|}
\hline & & \multicolumn{2}{|c|}{$\overline{\text { Best Result }}$} & \multicolumn{2}{|c|}{ Mean Result } & \multicolumn{2}{|c|}{ Worst Result } \\
\hline Problem & Optimal & SES & $\overline{\text { SR }}$ & SES & SR & SES & SR \\
\hline TF1 & -15 & -15.000000 & -15.000 & -15.000000 & -15.000 & -15.000000 & -15.000 \\
\hline TF2 & 0.803619 & 0.803569 & 0.803515 & 0.769612 & 0.781975 & 0.702322 & 0.726288 \\
\hline TF3 & 1 & 1.004329 & 1.000 & 1.003563 & 1.000 & 1.002604 & 1.000 \\
\hline TF4 & -30665.539 & -30665.539062 & -30665.539 & -30665.539062 & -30665.539 & -30665.539062 & -30665.539 \\
\hline TF5 & -6961.814 & -6961.813965 & -6961.814 & -6961.813965 & -6875.940 & -6961.813965 & -6350.262 \\
\hline TF6 & 24.306 & 24.313972 & 24.307 & 24.418837 & 24.374 & 24.560797 & 24.642 \\
\hline TF7 & 0.095825 & 0.095826 & 0.095825 & 0.095784 & 0.095825 & 0.095473 & 0.095825 \\
\hline TF8 & 680.63 & 680.669189 & 680.630 & 680.809829 & 680.656 & 681.199646 & 680.763 \\
\hline TF9 & 7049.25 & 7057.044434 & 7054.316 & 10771.416895 & 7559.192 & 16375.266602 & 8835.655 \\
\hline TF10 & 0.75 & 0.749018 & 0.750 & 0.749179 & 0.750 & 0.750647 & 0.750 \\
\hline TF11 & 1 & 1.000000 & 1.000000 & 1.000000 & 1.000000 & 1.000000 & 1.000000 \\
\hline TF12 & 0.053950 & 0.053964 & 0.053957 & 0.264135 & 0.057006 & 0.544346 & 0.216915 \\
\hline
\end{tabular}

Table 5: Comparison of results between our approach (SES) and Stochastic Ranking (SR) [13].

\begin{tabular}{|c|c|c|c|c|c|c|c|}
\hline & & \multicolumn{2}{|c|}{ Best Result } & \multicolumn{2}{|c|}{ Mean Result } & \multicolumn{2}{|c|}{ Worst Result } \\
\hline Problem & Optimal & SES & ASCHEA & SES & ASCHEA & SES & ASCHEA \\
\hline TF1 & -15 & -15.000000 & -15.0 & -15.000000 & -14.84 & -15.000000 & $N A$ \\
\hline TF2 & 0.803619 & 0.803569 & 0.785 & 0.769612 & 0.59 & 0.702322 & $N A$ \\
\hline TF3 & 1 & 1.004329 & 1.0 & 1.003563 & 0.99989 & 1.002604 & $\overline{N A}$ \\
\hline TF4 & -30665.539 & -30665.539062 & 30665.5 & -30665.539062 & 30665.5 & -30665.539062 & $\overline{N A}$ \\
\hline TF5 & -6961.814 & -6961.813965 & -6961.81 & -6961.813965 & -6961.81 & -6961.813965 & $\overline{N A}$ \\
\hline TF6 & 24.306 & 24.313972 & 24.3323 & 24.418837 & 24.66 & 24.560797 & $N A$ \\
\hline TF7 & 0.095825 & 0.095826 & 0.095825 & 0.095784 & 0.095825 & 0.095473 & $N A$ \\
\hline TF8 & 680.63 & 680.669189 & 680.630 & 680.809829 & 680.641 & 681.199646 & $N A$ \\
\hline TF9 & 7049.25 & 7057.044434 & 7061.13 & 10771.416895 & 7193.11 & 16375.266602 & $N A$ \\
\hline TF10 & 0.75 & 0.749018 & 0.75 & 0.749179 & 0.75 & 0.750647 & $N A$ \\
\hline TF11 & 1 & 1.000000 & $N A$ & 1.000000 & $N A$ & 1.000000 & $N A$ \\
\hline TF12 & 0.053950 & 0.053964 & $N A$ & 0.264135 & $N A$ & 0.544346 & $N A$ \\
\hline
\end{tabular}

Table 6: Comparison of results between our approach (SES) and ASCHEA [5]. NA = Not Available.

sign and Manufacture (ACDM 2002), volume 5, pages 273284, University of Exeter, Devon, UK, April 2002. SpringerVerlag.

[3] K. Deb. An Efficient Constraint Handling Method for Genetic Algorithms. Computer Methods in Applied Mechanics and Engineering, 186(2/4):311-338, 2000.

[4] M. Gorges-Schleuter, I. Sieber, and W. Jakob. Local interaction evolution strategies for design optimization. In 1999 Congress on Evolutionary Computation, pages 2167-2174, Piscataway, NJ, 1999. IEEE Service Center.

[5] S. B. Hamida and M. Schoenauer. ASCHEA: New Results Using Adaptive Segregational Constraint Handling. In Proceedings of the Congress on Evolutionary Computation 2002 (CEC'2002), volume 1, pages 884-889, Piscataway, New Jersey, May 2002. IEEE Service Center.

[6] J. Horn, N. Nafpliotis, and D. E. Goldberg. A Niched Pareto Genetic Algorithm for Multiobjective Optimization. In Proceedings of the First IEEE Conference on Evolutionary Computation, IEEE World Congress on Computational Intelligence, volume 1, pages 82-87, Piscataway, New Jersey, June 1994. IEEE Service Center.

[7] F. Jiménez and J. L. Verdegay. Evolutionary techniques for constrained optimization problems. In H.-J. Zimmermann, editor, 7th European Congress on Intelligent Techniques and Soft Computing (EUFIT'99), Aachen, Germany, 1999. Verlag Mainz. ISBN 3-89653-808-X.

[8] S. Koziel and Z. Michalewicz. Evolutionary Algorithms, Homomorphous Mappings, and Constrained Parameter Optimization. Evolutionary Computation, 7(1):19-44, 1999.
[9] E. Mezura-Montes and C. A. Coello Coello. A Simple Evolution Strategy to Solve Constrained Optimization problems. In Proceedings of Genetic and Evolutionary Computation Conference(GECCO'2003), pages 641-642, Heidelberg, Germany, July 2003. Chicago, Illinois, USA, SpringerVerlag. Lecture Notes in Computer Science No. 2723.

[10] Z. Michalewicz. Genetic Algorithms + Data Structures = Evolution Programs. Springer-Verlag, third edition, 1996.

[11] Z. Michalewicz and M. Schoenauer. Evolutionary Algorithms for Constrained Parameter Optimization Problems. Evolutionary Computation, 4(1):1-32, 1996.

[12] J. T. Richardson, M. R. Palmer, G. Liepins, and M. Hilliard. Some Guidelines for Genetic Algorithms with Penalty Functions. In J. D. Schaffer, editor, Proceedings of the Third International Conference on Genetic Algorithms (ICGA-89), pages 191-197, San Mateo, California, June 1989. George Mason University, Morgan Kaufmann Publishers.

[13] T. P. Runarsson and X. Yao. Stochastic Ranking for Constrained Evolutionary Optimization. IEEE Transactions on Evolutionary Computation, 4(3):284-294, September 2000.

[14] H. P. Schwefel. Evolution and Optimal Seeking. John Wiley \& Sons Inc., New York, 1995.

[15] A. E. Smith and D. W. Coit. Constraint Handling Techniques-Penalty Functions. In T. Bäck, D. B. Fogel, and Z. Michalewicz, editors, Handbook of Evolutionary Computation, chapter C 5.2. Oxford University Press and Institute of Physics Publishing, 1997. 